\title{
Spinal Cord Stimulation for Treatment of Pain in a Patient with Post Thoracotomy Pain Syndrome
}

Jordan Graybill, MD, Till Conermann, MD, Jack Kabazie, MD, and Sunil Chandy, MD

From: The Western Pennsylvania Hospital Institute for Pain Management, Pittsburgh, PA

Dr. Graybill is an anesthesiology resident at the Western Pennsylvania Allegheny Health System.

Dr. Conerman is Attending Physician and anesthesiologist, pain management at Western Pennsylvania Hospital. Dr. Kabazie is Attending Physician, Program Director of Pain Management Fellowship, and a Board certified anesthesiologist, pain management, Western Pennsylvania Hospital.

Dr. Chandy is a pain management fellow, Western Pennsylvania Hospital.

Address correspondence: Jordan Graybill, MD

The Western Pennsylvania Hospital Institute for Pain Management 4800 Friendship Avenue Pittsburgh, PA 15224 E-mail: muthergoosezo@yahoo. com

Disclaimer: There was no external funding in the preparation of this manuscript. Conflict of interest: None.

Manuscript received: 01/11/2011

Revised manuscript received: 05/21/2011

Accepted for publication: 05/25/2011

Free full manuscript: www.painphysicianjournal.com
Post Thoracotomy Pain Syndrome (PTPS) is defined as pain that occurs or persists in the area of the thoracotomy incision for at least 2 months following the initial procedure. The true incidence of PTPS is hard to define as literature reports a wide range of occurrence from $5 \%$ to $90 \%$. Thoracotomy is associated with a high risk of severe chronic postoperative pain. Presenting symptoms include both neuropathic pain in the area of the incision, as well as myofascial pain commonly in the ipsilateral scapula and shoulder.

Pain management can be challenging in these patients. Multiple treatments have been described including conservative treatments with oral nonsteroidal anti-inflammatory drugs (NSAIDs); topically applied, peripherally acting drugs; neuromodulating agents; physical therapy; transcutaneous electrical nerve stimulation as well as more invasive treatments including intercostal nerve blocks, trigger point steroid injections, epidural steroid injections, radiofrequency nerve ablation, cryoablation, and one case report of spinal cord stimulation. Unfortunately, a portion of these patients will have persistent pain in spite of multiple treatment modalities, and in some cases will experience worsening of pain.

This case report describes the novel utility and complete resolution of symptoms with spinal cord stimulation (SCS) in treatment of a patient with persistent PTPS.

In the operating room, a percutaneous octet electrode lead was placed using sterile technique under fluoroscopic guidance and loss-of-resistance technique. The octet electrode lead was subsequently advanced with the aid of fluoroscopy to the level of the T3 superior endplate just right of midline. The patient's pain distribution was captured optimally with stimulation at this level. With the assistance of a neurosurgeon, the lead was anchored, tunneled, and connected to a generator, which was implanted over the right iliac crest. The patient tolerated the procedure well with no complications.

We report the successful use of SCS as well as complete resolution of symptoms at 4 months follow-up, in a patient with persistent PTPS, which was resistant to other modalities.

In conclusion, studies designed to evaluate the effectiveness of SCS for PTPS may be warranted.

Key words: post thoracotomy pain syndrome, PTPS, chronic pain, spinal cord stimulation, thoracic pain, neuropathic pain, transcranial magnetic stimulation

Pain Physician 2011; 14:441-445
D ost Thoracotomy Pain Syndrome (PTPS) is defined as pain that occurs or persists in the area of the thoracotomy incision for at least 2 months following the initial procedure (1). The true incidence of PTPS is hard to define as the available literature reports a wide range of occurrence from $5 \%$ to $90 \%(2,3)$. Thoracotomy is associated with a high risk of severe chronic postoperative pain (4). 
Presenting symptoms include both neuropathic pain in the area of the incision, as well as myofascial pain commonly in the ipsilateral scapula and shoulder. Pain management can be challenging in these patients. Multiple treatments have been described including conservative treatments with oral nonsteroidal anti-inflammatory drugs (NSAIDs), topically applied peripherally acting drugs (lidocaine gel/patches, topical preparations of NSAIDs, salicylates, and capsaicin), neuromodulating agents, transcutaneous electrical nerve stimulation, and physical therapy. More invasive therapies have been described including intercostal nerve blocks, trigger point injections, epidural steroid injections, radiofrequency nerve ablation, cryoablation, and one case report of spinal cord stimulation (SCS) $(2,5)$. Unfortunately some of these patients will have persistent pain in spite of multiple treatment modalities, and in some cases will experience worsening of pain $(2,6)$. In such cases, SCS may be considered due to its effectiveness in neuropathic pain (7-10).

We describe the use of SCS for treatment of persistent pain that was resistant to other treatment modalities. In this case report, the patient had complete resolution of symptoms reported during the last follow-up office visit at 4 months post stimulator implantation.

\section{Case Report}

A 71-year-old male presented to our clinic with a 3-year history of persistent pain involving the right chest in the distribution of a thoracotomy incision, following an esophagectomy for cancer. The patient characterized the pain as constant, and burning in nature with associated allodynia. The pain was aggravated by light touch and pressure, which prevented the patient from sleeping. He rated the pain $8 / 10$ on the verbal numeric rating scale of 0 to 10 . Previous workups included a bone scan, CT scan of the chest, chest x-ray, and thorough physical examinations, ruling out metastatic disease and other pathology (see discussion). Prior medical treatments included oral morphine, and hydrocodone/ acetaminophen 10/500, which allowed the patient to sleep, but prevented him from participating in daily activities secondary to the associated sedation and lethargy. On physical examination the patient had significant allodynia, hyperesthesia, and hyperalgesia covering a large area of the lateral right chest wall from the posterior axillary line to the right parasternal area in the distribution of the thoracotomy scar. The scar was well healed with no obvious neuroma formation, swelling, or signs of infection. A computed tomography scan showed degenerative changes within the cervical spine, and bilateral shoulders. A whole body bone scan showed no scintigraphic evidence of osseous metastatic disease. The patient was started on a gabapentin titration schedule, and was continued on morphine ER $30 \mathrm{mg}$ twice a day and hydrocodone/acetaminophen 10/500 mg 1-2 tabs 3 times a day for breakthrough pain relief. The patient was scheduled and received 3 thoracic epidural injections of $120 \mathrm{mg}$ of methylprednisolone and $7.5 \mathrm{mg}$ of bupivicaine at the T7-8 vertebral level. The gabapentin was ultimately discontinued secondary to a lack of desired efficacy and associated oversedation. On follow-up, the patient described minimal relief of symptoms after 3 thoracic epidural steroid/local anesthetic injections in addition to oral opioids.

Hoping to achieve improved pain control and associated improvement in his level of functioning, the patient was offered a trial of SCS. The risks of the procedure, including bleeding, infection, headache, nerve injury, and treatment failure, were reviewed. After informed consent was obtained, the patient was taken to the operating room and placed in a prone position. His back was prepped and draped in a normal sterile fashion. The skin and subcutaneous tissues were infiltrated with approximately $5 \mathrm{~mL}$ of $1 \%$ preservative free lidocaine at the needle entry site. Under fluoroscopic guidance, a 14-gauge Tuohy needle was advanced using the loss-of-resistance technique at the T12-L1 right paramedian interspace. After entering the epidural space, placement was confirmed with fluoroscopy and a percutaneous octet electrode lead was subsequently advanced with the aid of fluoroscopy to the level of the T3 inferior endplate just right of midline (Fig. 1). The patient's pain distribution was captured optimally with stimulation at this level. The lead was secured to the back using benzoin tincture, steri-strips, and then covered with a Tegaderm dressing. The patient tolerated the procedure well. Discharge and operational instructions for the external generator were provided.

During the 5-day outpatient trial, the patient experienced excellent pain relief with a reported pain score of $0 / 10$ on the verbal pain scale. He required only minimal breakthrough medications. During the trial period the lead was not repositioned. The patient was offered permanent implantation of the stimulator. The patient was again taken to the operating room, and the abovedescribed procedure was replicated with the first electrode contact positioned at the T3 superior endplate just right of midline (Fig. 2). During this implant the patient reported the best capture of his symptoms at the 


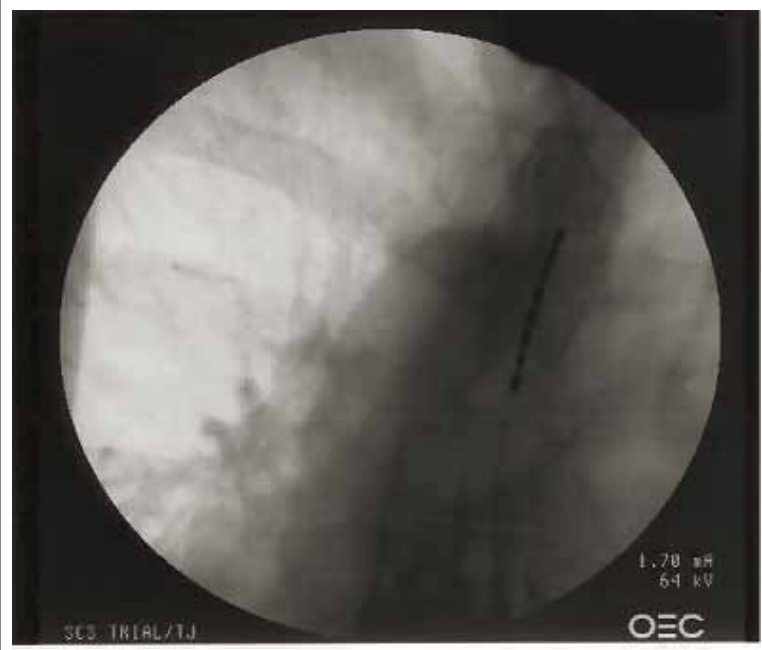

Fig. 1. PA view of the octet electrode lead at the level of the T3 vertebral body inferior endplate, just right of midline.

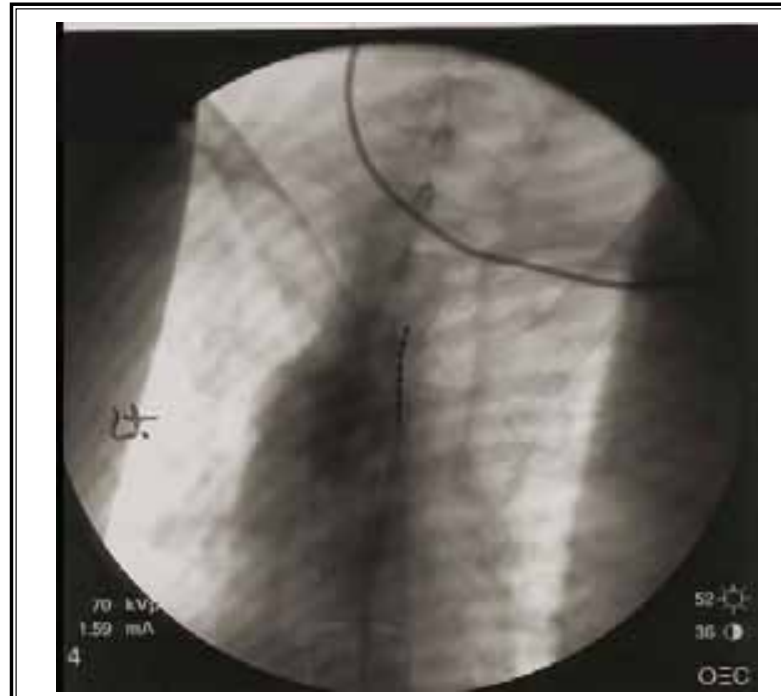

Fig. 2. PA view of the octet electrode lead at the level of the T3 vertebral body superior endplate, just right of midline. superior endplate of $\mathrm{T} 3$, with an amplitude of $6.9 \mathrm{~mA}$, pulse with of $670 \mu \mathrm{S}$, and frequency of $60 \mathrm{~Hz}$. With the assistance of a neurosurgeon, the lead was anchored, tunneled, and connected to a generator, which was implanted over the right iliac crest. The patient tolerated the procedure well with no complications.

The patient was seen one week postoperatively. At that time he had only minimal incisional pain with complete resolution of his right chest wall pain, and was able to perform all daily activities without impairment. Although the patient was initially scheduled for follow-up in 2 months, he subsequently cancelled this appointment, reporting complete resolution of his previous symptoms. At an office follow-up 3 months after the implantation, he continued to report no pain in the area of his thoracotomy, as well as resolution of his incisional pain. The patient reported that during charging of the generator and while driving (with the SCS turned off), he had return of pain (9/10 verbal numeric pain scale); however, the patient had not had the SCS turned off for extended periods besides the above. He was reporting complete resolution of his symptoms and that his level of activity remains significantly improved and without limitations. At 4 months after subpectoral implantation, the patient's generator settings were adjusted to an amplitude of $8.8 \mathrm{~mA}$, pulse width of $390 \mu \mathrm{S}$, and a frequency of $50 \mathrm{~Hz}$. He again reported complete resolution of his symptoms.

\section{Discussion}

PTPS was first described in 1944 by 2 army surgeons (11). PTPS is defined as pain that recurs or persists at the incision site or in the dermatomal distribution of the intercostal nerves for longer than 2 months after a thoracotomy. Thoracotomy is regarded as one of the most painful surgical procedures performed (4). The incidence of PTPS varies from $5 \%$ up to $90 \%$ in the literature $(2,3)$. This wide range of occurrence is attributed to different definitions used to describe and assess pain, lack of large prospective studies, small sample size, varying surgical techniques, nonstandardized perioperative management, and varying periods of follow-up. Nonetheless, most studies report the overall incidence at over $40 \%$, with severe disabling pain persisting in up to $5 \%$ of patients (12). Patients experiencing PTPS will commonly present with allodynia, hyperalgesia, hypoesthesia, dysesthesia, abnormal sudomotor activity, pleuritic pain, and focal tenderness over the area of the incision. This pain is often aggravated by deep breathing and movement of the ipsilateral shoulder (2). The neuropathic component of pain is attributed to direct intercostal nerve injury from spreading of the ribs, direct surgical trauma, suture entrapment of the nerve, and/or local inflammation, and scar tissue $(2,13,14)$.

Diagnosis is usually that of exclusion, as pathology from multiple sources can cause symptoms similar to 
PTPS. Pathology that should be ruled out includes intercostal neuroma, rib fractures, infection, costochondral dislocation, tumor recurrence, and costochondritis. A workup generally includes a thorough physical examination, chest radiograph and computed tomography. Performing an intercostal nerve block can generally identify intercostal neuralgia, which is the most commonly implicated cause of PTPS (2).

Treatment of PTPS is multimodal and often aimed at preemptive analgesia in an attempt to minimize sensitization and a windup phenomenon. It has been shown that predictive factors for developing PTPS include severe acute postoperative pain, as well as being female $(3,6,14)$. Thoracic epidural has become widely used for acute postoperative pain control in patients receiving a thoracotomy. Cryoablation of intercostal nerves was commonly performed during surgery, however has fallen out of favor as it has been associated with an increased incidence of neuropathic pain in some studies (15). After surgery therapy is aimed at rehabilitation. Physical therapy is important for early shoulder mobilization, as shoulder dystocia has been reported in over $75 \%$ of thoracotomy patients (16). In addition, early scar mobilization can diminish the incidence of PTPS by reducing adherence to chest wall structures, underlying nerve entrapment, and range of motion restriction of the shoulder.

Once PTPS presents, a multimodal array of treatments exist including NSAIDs, topically applied peripherally acting drugs (lidocaine gel/patches, topical preparations of NSAIDs, salicylates, and capsaicin), cognitive behavior treatments, neuromodulators such as gabapentin, intercostal nerve blocks, epidural steroid injections, thoracic nerve root blocks, and radiofrequency ablation (17-19). Long-term treatment options described include intrathecal opioid pumps, SCS, and peripheral nerve stimulation $(7-10,20)$. The mechanism of action in SCS is not fully understood at this time. Theories as to the exact mechanism include the "gate control theory" first described in 1965 which speculated that nerves carrying painful peripheral stimuli and nerves carrying touch and vibratory sensation both terminate in the dorsal horn. It is hypothesized that creating a vibration sensation with the SCS could manipulate and "close the gate" to the pain sensation (21). Other theories include central desensitization, and inhibition of phenotypic switching in A-beta fibers (22). It is likely that in this specific case report if the SCS were to be turned off for an extended period of time, a portion of the original neuropathic pain would recur. At this time there are no studies comparing conservative to interventional treatment as invasive therapies are reserved for patients who have failed conservative treatment. Data for supporting one interventional treatment option over another is limited as there are no studies comparing these interventions. Intercostal nerve blocks, radiofrequency ablation, steroid injections, and thoracic nerve root blocks are temporary and often have to be repeated multiple times and possibly indefinitely and thus cannot be compared to SCS which is considered more permanent. Despite the preemptive and postoperative treatment of pain, PTPS often remains severe in a portion of these patients.

A possible future treatment option for this syndrome, as well as other chronic pain conditions, involves transcranial magnetic stimulation (TMS). TMS has been described since the early 1990s for the possible treatment of many psychological diseases such as depression, chronic pain, Parkinson disease, anxiety, and schizophrenia. TMS creates a magnetic field which is transformed at the level of the cerebral cortex to an electric field, which acts on neurons transynaptically at the level of tangentially aligned interneurons. The exact mechanism is not fully understood at this time, but it is thought that TMS is able to manipulate some of the changes in the central nervous system seen secondary to chronic pain, thus possibly reversing some neuroplastic changes seen in the above mentioned diseases (23). Transcranial mapping has also been described using TMS through a process of applying TMS and simultaneous motor evoked potentials. Measuring changes in amplitude and location of signals during this process makes it possible to achieve brain cartography (24). This tool may prove useful in locating areas of central neuroplastic change in the cortex.

\section{Conclusion}

The prevalence of post thoractomy pain syndrome is as high as $90 \%$, with severe debilitating symptoms occurring in up to $5 \%$ of this population. In our literature search, only one source was found describing SCS for PTPS (5), although it was mentioned as a treatment option in multiple sources. Based on our clinical experience, and given the limited clinical success of other reported conservative as well as interventional treatment measures, in a subset of patients with persistent severe PTPS, SCS should be considered as a viable long-term treatment option. Studies designed to evaluate the effectiveness of SCS for PTPS may be warranted. 


\section{References}

1. Merskey $\mathrm{H}$. Classification of chronic pain: Description of chronic pain syndromes and definitions of pain terms. Pain 1986; 3:S138-S139

2. Karmaker M, Ho A. Postthoracotomy pain syndrome. Thoracic Surg Clin 2004; 14:345-352.

3. Erdek M, Staats PS. Chronic pain after thoracic surgery. Thorac Surg Clin 2005; 15:123-130.

4. Kehlet H, Jensen TS, Woolf CJ. Persistent postsurgical pain: Risk factors and prevention. Lancet 2006; 367:1618-1625.

5. Segal R, Stacey BR, Rudy TE, Baser S, Markham J. Spinal cord stimulation revisited. Neurol Res 1998; 20:391-396.

6. Gotoda Y, Kambara N, Sakai T, Kishi Y, Kodama K, Koyama T. The morbidity, time course, and predictive factors for persistent post-thoracotomy pain. Eur J Pain 2001; 5:89-96

7. Manchikanti L, Boswell MV, Singh $V$, Benyamin RM, Fellows B, Abdi S, Buenaventura RM, Conn A, Datta S, Derby R, Falco FJE, Erhart S, Diwan S, Hayek SM, Helm S, Parr AT, Schultz DM, Smith HS, Wolfer LR, Hirsch JA. Comprehensive evidence-based guidelines for interventional techniques in the management of chronic spinal pain. Pain Physician 2009; 12:699-802.

8. Frey ME, Manchikanti L, Benyamin RM, Schultz DM, Smith HS, Cohen SP. Spinal cord stimulation for patients with failed back surgery syndrome: A systematic review. Pain Physician 2009; 12:379397.

9. Manchikanti L, Boswell MV, Datta S, Fellows B, Abdi S, Singh V, Benyamin RM, Falco FJE, Helm S, Hayek S, Smith
HS. Comprehensive review of therapeutic interventions in managing chronic spinal pain. Pain Physician 2009; 12:E123E198.

10. Manchikanti L, Datta S, Gupta S, Munglani R, Bryce DA, Ward SP, Benyamin RM, Sharma ML, Helm II S, Fellows B, Hirsch JA. A critical review of the American Pain Society clinical practice guidelines for interventional techniques: Part 2. Therapeutic interventions. Pain Physician 2010; 13:E215-E264.

11. Blades B, Dugan DJ. War wounds of the chest. J Thorac Surg 1944; 13:294-306.

12. Maguire MF, Ravenscroft A, Beggs D, Duffy J. A questionnaire study investigating the prevalence of the neuropathic component of chronic pain after thoracic surgery. Eur J Cardiothorac Surg 2006; 29:800-805.

13. Hazelrigg SR, Cetindag IB, Fullerton J. Acute and chronic pain syndromes after thoracic surgery. Surg Clin North Am 2002; 82:849-865

14. Perttunen K, Tasmuth T, Kalso E. Chronic pain after thoracic surgery: A follow up study. Acta Anaesthesiol Scand 1999; 43:563-567

15. Ju H, Feng Y, Yang B, Wang J. Comparison of epidural analgesia and intercostal nerve cryoanalgesia for post-thoracotomy pain control. Eur J Pain 2008; 12:378384 .

16. Gerner, P. Post thoracotomy pain management problems. Anesthesiol Clin 2008; 26:355-367, vii.

17. Sihoe AD, Lee TW, Wan IYP, Thung KH, Yim APC. The use of gabapentin for postoperative and post-traumatic pain in thoracic surgery patients. Eur ] Cardio- thorac Surg 2006; 29:795-799

18. Atluri S, Datta S, Falco FJ, Lee M. Systematic Review of Diagnostic Utility and Therapeutic Effectiveness of Thoracic Facet Joint Interventions. Pain Physician 2008;11;611-629.

19. Manchikanti L, Cash KA, McManus CD, Pampati V, Benyamin RM. A preliminary report of a randomized double-blind, active controlled trial of fluoroscopic thoracic interlaminar epidural injections in managing chronic thoracic pain. Pain Physician 2010; 13:E357-E369.

20. Falco FJE, Erhart S, Wargo BW, Bryce DA, Atluri S, Datta S, Hayek SM. Systematic review of diagnostic utility and therapeutic effectiveness of cervical facet joint interventions. Pain Physician 2009; 12:323-344.

21. Melzack R, Wall PD. Pain mechanisms: A new theory. Science 1965; 150:971-979.

22. Linderoth B, Foreman R. Mechanisms of spinal cord stimulation in painful syndromes: Role of animal models. Pain Medicine 2006: 7:14-26.

23. Guipponi G, Pycha R, Dell'Osso B, Pompili $M$, Walpoth $M$, Hausmann A, DiPauli J, Erfurth A, Conca A. Neurophysiological and neuropsychiatric aspects of transcranial magnetic stimulation. Clinical Neuropsychiatry 2009; 6:234-245.

24. Brasil-Neto JP, McShane LM, Fuhr P, Hallet $M$ \& Cohen LG. Topographic mapping of the human motor cortex with magnetic stimulation: factors affecting accuracy and reducibility. Electroencephalogr Clin Neurophysiol 1992; 85:9-16. 
